\title{
Evaluation of a novel monoclonal enzyme immunoassay for detection of Helicobacter pylori antigen in stool from children
}

\author{
S Koletzko, N Konstantopoulos, D Bosman, A Feydt-Schmidt, A van der Ende, \\ N Kalach, J Raymond, H Rüssmann
}

See end of article for authors' affiliations

Correspondence to:

Dr S Koletzko, Kinderklinik

and Kinderpoliklinik im Dr

$\checkmark$ Haunersches

Kinderspital,

Ludwig-Maximilians-University

Munich, Pettenkoferstr 8a,

D-80336 Munich,

Germany;

Koletzko@pk-i.med.

uni-muenchen.de

Accepted for publication 26 November 2002
Background: Reliable non-invasive methods for detection of Helicobacter pylori infection are required to investigate the incidence, transmission, and clearance of infection in childhood.

Aim: To evaluate a new monoclonal enzyme immunoassay (EIA) (FemtoLab H pylori $\mathrm{Cnx}$ ) for detection of $H$ pylori antigen in stool in a large cohort of children compared with invasive diagnostic methods and the ${ }^{13} \mathrm{C}$ urea breath test.

Patients and methods: A total of 302 symptomatic previously untreated children laged 0.5-18.7 years; 148 girls) were recruited at three centres. H pylori status was defined by results of culture, histology, the rapid urease test, and the ${ }^{13} \mathrm{C}$ urea breath test. Stool samples were investigated locally by the EIA using two different production lots. According to the manufacturer's recommendations, an optical density (OD) of 0.150 was used as a cut off value.

Results: OD values clearly differentiated between the $92 \mathrm{H}$ pylori infected and the 210 non-infected children (median (5th-95th percentiles) 2.729 (0.232->4.000) v 0.021 (0.009-0.075)). Only two false positive and two false negative results occurred, giving a sensitivity, specificity, positive predictive value, and negative predictive value of $98 \%, 99 \%, 98 \%$, and $99 \%$, respectively. No significant relation was found between age and OD values in infected or non-infected children.

Conclusions: The monoclonal stool antigen EIA was excellent in diagnosing $H$ pylori infection in symptomatic children. Accuracy was independent of the laboratory, production lot used, or the child's age. Because only $18 / 116$ children $<6$ years of age were infected with $H$ pylori, further validation of the test is needed in young infected children.
Helicobacter pylori infection is almost always acquired in early childhood and usually persists throughout life unless a specific treatment is given. To investigate transmission, incidence, spontaneous clearance, and preventive measures in $H$ pylori infection, non- invasive tests are required which must be reliable in all age groups, including toddlers, in which the incidence of new infections is highest. ${ }^{2}$ Serological tests show a low sensitivity in young children; also, serology cannot be considered a non-invasive test in children. ${ }^{3-5}$ The ${ }^{13} \mathrm{C}$ urea breath test (UBT) has an excellent sensitivity regardless of age but specificity decreases in infants and young children..$^{5-7}$ In addition, in this age group UBT requires trained staff for air sampling with a face mask, which may be unpleasant for the child. An enzyme immunoassay (EIA) to detect $H$ pylori antigen in stool would circumvent these difficulties as stool samples can be obtained from children without their active collaboration. In addition, EIA tests do not require expensive instruments such as a mass spectrometer for UBT. Much experience has been gained with the HpSA (Meridian Diagnostics, Cincinnati, USA) which uses polyclonal antibodies to detect antigens in stool. ${ }^{89}$ Overall performance in diagnosing $H$ pylori infection or evaluating the success of eradication therapy has been good ${ }^{10-12}$ but some limitations and discrepancies have been reported with respect to inter charge variability, cut off values, and lower accuracy after eradication therapy. ${ }^{13-15}$ The FemtoLab H pylori Cnx is a novel test based on monoclonal antibodies to detect $H$ pylori antigen in faeces. Preliminary results using developmental test kits have been reported in children. ${ }^{13}$ The aim of this prospective multicentre study was to evaluate the monoclonal EIA for detection of $H$ pylori infection in a large number of children compared with well defined $H$ pylori status established by invasive diagnostic techniques and UBT.

\section{PATIENTS AND METHODS}

Patients

Over two years, 302 unselected children (148 girls, 154 boys) with abdominal symptoms were enrolled in three paediatric hospitals (Munich n= 173; Amsterdam n=97; Paris n=32). All children underwent upper gastrointestinal endoscopy because of abdominal symptoms suggestive of organic disease. In all 302 children, stool test results were compared with $H$ pylori status defined by the results of biopsy based methods (rapid urease test, culture, and histology) and/or UBT.

Children were excluded if they had taken antibiotic or acid suppressive drugs (proton pump inhibitors, $\mathrm{H}_{2}$ receptor antagonists, antacids, bismuth preparations) within four weeks prior to testing, if they had received previous anti$H$ pylori therapy, or if the $H$ pylori status was not clearly defined. The study was approved by the local ethics committees, and informed consent was obtained from the parents and children, if appropriate.

\section{Definition of $\boldsymbol{H}$ pylori status}

During upper endoscopy, two biopsies each from the gastric antrum and corpus were taken for histological examination, formalin fixed, stained with haematoxylin-eosin and modified

Abbreviations: EIA, enzyme immunoassay; UBT, ${ }^{13} \mathrm{C}$ urea breath test; OD, optical density. 
Table 1 Performances of the monoclonal stool antigen test according to age group, and for all children

\begin{tabular}{lcccccc}
\hline Age group $(\mathrm{y})$ & $\mathrm{n}(\mathrm{Hp}+)$ & Sensitivity & Specificity & Accuracy & PPV & NPV \\
\hline$<6$ & $116(18)$ & $94.4[72.7-99.9]$ & $98.0[92.8-99.8]$ & $97.4[92.6-99.5]$ & $89.5[66.9-98.7]$ & $99.0[94.4-99.9]$ \\
$\geqslant 6-<12$ & $106(42)$ & $97.6[87.4-99.9]$ & $100[94.4-100]$ & $99.1[94.9-99.9]$ & $100[91.4-100]$ & $98.5[91.7-99.9]$ \\
$\geqslant 12-<18$ & $80(32)$ & $100[89.1-100]$ & $100[92.6-100]$ & $100[95.5-100]$ & $100[89.1-100]$ & $100[92.6-100]$ \\
All children & $302(92)$ & $97.8[92.4-99.7]$ & $99.0[96.6-99.9]$ & $98.7[96.6-99.6]$ & $97.8[92.4-99.7]$ & $99.0[96.6-99.9]$ \\
\hline
\end{tabular}

PPV, positive predictive value; NPV, negative predictive value.

$\mathrm{Hp}+$, Helicobacter pylori positive.

Values are median [5th-95th percentiles]

Giemsa, and viewed for the presence of $H$ pylori by a local pathologist who was blinded to the results of the other tests $(\mathrm{n}=302)$. One antral specimen each was obtained for the rapid urease test $(n=222)$ and for bacterial culture $(n=309)$. Biopsies were transported to the local microbiological laboratory in transport media and processed within four hours. UBT $(\mathrm{n}=274)$ was performed according to a standardised protocol, as described previously. ${ }^{6}$ The test was defined as positive when changes from baseline values after 30 minutes were $\geqslant 5 \%$.

$H$ pylori status was defined as positive if culture and/or at least two of the other methods (histology, rapid urease test, UBT) gave positive results. A negative $H$ pylori status was considered if all tests gave concordant negative results.

\section{Stool antigen test}

Parents of children scheduled for endoscopy were asked to bring a stool sample of their child at the time of the procedure or to send it in by mail within three days after endoscopy before any therapy was initiated. Samples were stored at $-20^{\circ} \mathrm{C}$ until analysed.

The stool antigen test (FemtoLab H pylori Cnx (Martinsried, Germany), identical to HpStAR (DakoCytomation GmbH, Hamburg, Germany) and Ridascreen FemtoLab (RBiopharm AG, Darmstadt, Germany) was performed according to the manufacturer's recommendations at the three local microbiological laboratories using two different production lots. Those performing and reading the test were unaware of the $H$ pylori status of the children tested. The stool antigen test is an EIA which uses monoclonal mouse anti-H pylori antibodies adsorbed to microwells as capture antibody. Firstly, $50 \mu \mathrm{l}$ of supernatant of the diluted stool sample $(0.1 \mathrm{~g}$ stool in $0.5 \mathrm{ml}$ sample diluent) and thereafter $50 \mu \mathrm{l}$ conjugated monoclonal antibody solution were added to the wells and incubated for one hour at room temperature on a shaker. Unbound material was removed by washing four times with a washing buffer. After washing, $100 \mu \mathrm{l}$ of a substrate solution were added and incubated for 10 minutes. After addition of $100 \mu \mathrm{l}$ of a stopping solution, the results were read by spectrophotometry (450/630 nm double wavelength).

According to the manufacturer's guidelines, an optical density (OD) $<0.150$ was defined as a negative and an $\mathrm{OD} \geqslant 0.150$ as a positive test result.

\section{Statistics}

Sensitivity and specificity with confidence intervals, and positive and negative predictive values of the stool test were calculated against the defined $H$ pylori status as gold standard. Statistical analysis was performed using SPSS (Statistical Package for Social Sciences version 9.1; SPSS Inc., Chicago, Illinois, USA). Correlation between age and OD values was analysed by the Spearman-Rho test. The likelihood ratios for a positive and negative test result were determined. ${ }^{16}$

\section{RESULTS}

According to the predefined criteria, 92 (30.5\%) of 302 patients were $H$ pylori positive; in 88/92 children, culture was positive for $H$ pylori. The remaining 210 (69.5\%) children had

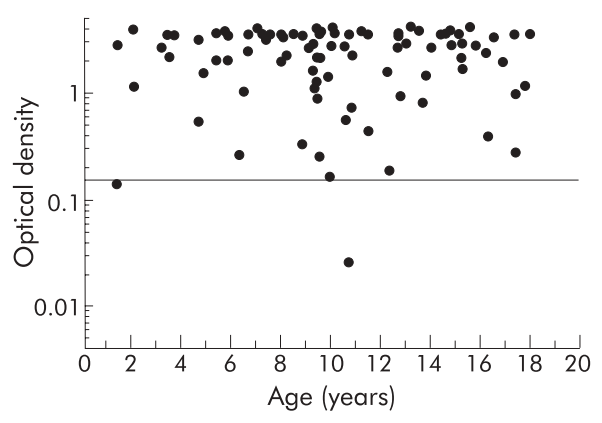

Figure 1 Optical density (OD) values in relation to age in 92 children with a positive Helicobacter pylori status. The cut off value was set at an OD of 0.150 .

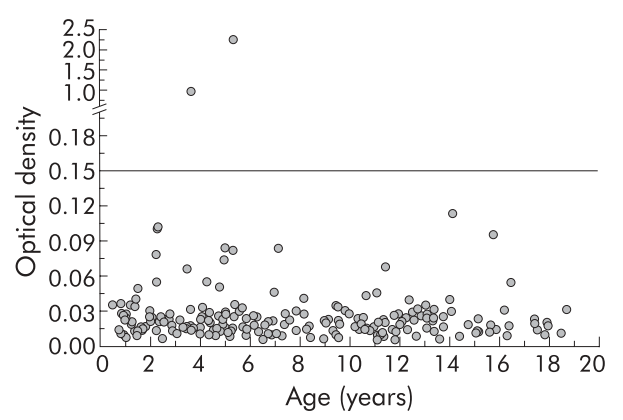

Figure 2 Optical density (OD) values on a log scale in relation to age in 210 children with a negative Helicobacter pylori status. The cut off value was set at an OD of 0.150 .

negative results in all diagnostic tests performed and were therefore considered $H$ pylori negative.

Age of the children ranged from 0.5 to 18.7 years. A total of 116 patients were $<6$ years of age ( 18 were positive and 98 negative for $H$ pylori), 106 were $\geqslant 6-<12$ years ( 42 positive, 64 negative), and the remaining 80 children were $\geqslant 12$ years of age ( 32 positive, 48 negative) (table 1 ). The proportions of infected to non-infected children in the three centres were as follows: in Munich, 55 to 118; in Amsterdam, 27 to 70; and in Paris, 10 to 22. The geographical background of the children's families was Northern or Western Europe in 188 cases, 23 families came from Southern Europe, three from Eastern Europe, 45 from Turkey, nine from Asia, 27 from Africa, and seven from America.

OD values in the EIA clearly differentiated between $H$ pylori infected (median 2.729 (5th-95th percentiles 0.232->4.000)) and non-infected children $(0.021(0.009-0.076))$. No significant correlation was seen between age of the patient and OD values in the $H$ pylori positive $(r=-0.077, \mathrm{p}=0.265)$ or negative $(r=-0.024, \mathrm{p}=0.821)$ groups (figs 1,2$)$.

Four children were misclassified by the stool antigen test. Two patients from Munich had false positive results; one was infected by Campylobacter jejunii at the time of endoscopy. The two patients with false negative results came from Amsterdam; both had positive bacterial cultures. One of the four false 
results had an OD value that was close to the cut off value (fig 1). Sensitivity, specificity, accuracy, and positive and negative predictive values (with 95\% confidence intervals) are presented in table 1 according to the three different age groups and globally for the total cohort. The likelihood ratio for a positive test result was calculated as 103 .

\section{DISCUSSION}

To the best of our best knowledge, this is the first prospective multicentre based study of a novel monoclonal EIA stool test used to establish a diagnosis of $H$ pylori infection in children. For every child, $H$ pylori status was assessed using three different tests. In fact, culture, which is considered to be $100 \%$ specific, was successful in $88 / 92$ children with a positive $H$ pylori status. The monoclonal EIA on stool samples correctly classified 298 of 302 children, giving an accuracy of $98 \%$.

To date, only one study has been published using this monoclonal EIA in children prior to treatment. Makristathis et al used a developmental kit provided by the manufacturer at a time when the test was not yet marketed. ${ }^{13}$ The authors performed the test in 79 children, 39 of whom were considered to be $H$ pylori positive according to positive results from UBT and serology. The test yielded a sensitivity of $98 \%$ and a specificity of $97 \%$, which is similar to our results of $98 \%$ and $99 \%$, respectively. In our study, these excellent results were obtained in spite of the fact that the test was performed in three different laboratories using two different production lots. In contrast, the HpSA, which is of polyclonal origin, seems to have problems with lot to lot variability. ${ }^{13}$ This variability is reflected by the wider range of sensitivity and specificity values, reported in some studies to be as low as 63\%, even in patients before therapy. ${ }^{8}$ In a recent multicentre European trial involving non-invasive tests in 316 children with a biopsy based $H$ pylori status, HpSA achieved a sensitivity of only $72.7 \% .^{17}$

Differentiation between positive and negative results (figs 1,2 ) is valuable. In contrast with the HpSA test, no grey zone is necessary. To improve the accuracy of the HpSA, some investigators have suggested adapting the cut off value. ${ }^{12} 1415$

In accordance with our previous experience with the polyclonal test, we did not find any relation between OD values and patient age. This is particular advantageous in paediatric settings as both serology and UBT are less accurate in younger children. ${ }^{35618}$ With respect to specificity, we are confident that the monoclonal EIA for detection of $H$ pylori antigen in stool is excellent in children younger than six years of age, as 98 of the non- infected children in our study belonged to this age group, and 47 of those were less than three years of age. In contrast, only $18 \mathrm{H}$ pylori infected children were less than six years of age and only four were less than three years of age. This is reflected by the larger $95 \%$ confidence intervals for sensitivity compared with specificity in this age group. Therefore, for final conclusions regarding sensitivity, more $H$ pylori infected infants and toddlers need to be studied with the monoclonal antigen test.

We cannot judge how the test will perform in developing countries with a high prevalence of diarrhoea in young children due to gastrointestinal infections. It is noteworthy that one of the two children with a false positive test result suffered from Campylobacter jejunii infection at the time of testing, indicating that there might be some cross reactivity between the two bacterial species. Also, we need to establish how acid suppressive drugs or recent intake of different antibiotics influence the test results. We excluded all patients who had taken any of these medications during the four weeks prior to testing to obtain a well defined $H$ pylori status, as both types of substances may suppress growth of $H$ pylori and cause false negative results. ${ }^{8}$

In conclusion, the monoclonal EIA stool test is easy to perform and provides excellent differentiation between positive and negative test results. In symptomatic children the test is well suited for evaluation of $H$ pylori status. The high accuracy seems to be independent of the laboratory, production lots, and age of the child. An age specific cut off value is not required, even in young children. Therefore, if further studies in children confirm our results, this test may become an excellent tool to study the incidence, spontaneous clearance of $H$ pylori infection, and effect of preventive measures such as vaccination.

\section{ACKNOWLEDGEMENT}

The study was supported by the Child Health Foundation, Munich. Connex $\mathrm{GmbH}$, Martinsried, Germany, provided free test kits for determination of stool antigen.

\section{Authors' affiliations}

S Koletzko, N Konstantopoulos, A Feydt-Schmidt, Kinderklinik and Kinderpoliklinik, Dr von Haunersches Kinderspital, Ludwig-Maximilians University, Munich, Germany

D Bosman, A van der Ende, Emma Children's Hospital, Amsterdam, the Netherlands

N Kalach, J Raymond, Hospital St Vincent de Paul, Paris, France

H Rüssmann, Max von Pettenkofer Institut,

Ludwig-Maximilians-University, Munich, Germany

\section{REFERENCES}

1 Rothenbacher D, Inceoglu J, Bode G, et al. Acquisition of Helicobacter pylori infection in a high-risk population occurs within the first 2 years of ife. J Pediatr 2000;136:744-8.

2 Thomas JE, Dale A, Harding $M$, et al. Helicobacter pylori colonization in early life. Pediatr Res 1999:45:218-23

3 Oliveira AMR, Rocha GA, Queiroz DM, et al. Evaluation of enzyme-linked immunosorbent assay for the diagnosis of Helicobacter pylori infection in children from different age groups with and without duodenal ulcer. J Pediatr Gastroenterol Nutr 1999;28:157-61.

4 Kindermann A, Konstantopoulos N, Lehn N, et al. Evaluation of two commercial enzyme immunoassays, testing immunoglobulin $G(\mathrm{lg} G)$ and lgA responses, for diagnosis of Helicobacter pylori infection in children. Clin Microbiol 2001;39:3591-6.

5 Koletzko S, Feydt-Schmidt A. Infants differ from teenagers: use of non-invasive tests for detection of Helicobacter pylori infection in children. Eur J Gastroenterol Hepatol 2001;13:1047-52.

6 Kindermann A, Demmelmair H, Koletzko B, et al. Influence of age on 13C-urea breath test results in children. J Pediatr Gastroenterol Nutr 2000:30:85-91.

7 Imrie C, Rowland M, Bourke B, et al. Limitations to carbon 13-labeled urea breath testing for Helicobacter pylori in infants. J Pediatr 2001;139:734-7

8 Gisbert JP, Pajares JM. Diagnosis of Helicobacter pylori infection by stool antigen determination: a systematic review. Am J Gastroenterol 2001.96:2829-38.

9 Kabir S. Detection of Helicobacter pylori in faeces by culture, PCR and enzyme immunoassay. J Med Microbiol 2001;50:1021-9.

10 Konstantopoulos N, Russmann H, Tasch C, et al. Evaluation of the Helicobacter pylori stool antigen test $(\mathrm{HpSA})$ for detection of Helicobacter pylori infection in children. Am J Gastroenterol 2001;96:677-83.

11 van Doorn OJ, Bosman DK, van'† Hoff BW, et al. Helicobacter pylori stool antigen test: a reliable non-invasive test for the diagnosis of Helicobacter pylori infection in children. Eur J Gastroenterol Hepatol 2001;13:1061-5.

12 Oderda G, Rapa A, Ronchi B, et al. Detection of Helicobacter pylori in stool specimens by non-invasive antigen enzyme immunoassay in children: multicentre Italian study. BM 2000;320:347-8.

13 Makristathis A, Barousch W, Pasching, et al. Two enzyme immunoassays and PCR for detection of Helicobacter pylori in stool specimens from pediatric patients before and after eradication therapy. $J$ Clin Microbiol 2000;38:3710-14

14 Leodolter A, Agha-Amiri K, Peitz U, et al. Validity of a Helicobacter pylori stool antigen assay for the assessment of $\mathrm{H}$. pylori status following eradication therapy. Eur J Gastroenterol Hepatol 2001 ; 13:673-6.

15 Forne M, Dominguez J, Fernandez-Banares F, et al. Accuracy of an enzyme immunoassay for the detection of Helicobacter pylori in stool specimens in the diagnosis of infection and posttreatment check-up. Am J Gastroenterol 2000;95:2200-5.

16 Jaeschke R, Guyatt G, Sackett DL. Users' guides to the medical literature. III. How to use an article about a diagnostic test. A. Are the results of the study valid? Evidence-Based Medicine Working Group. JAMA 1994;271:389-91.

17 Megraud F, on behalf of the Paediatric Task Force of $\mathrm{H}$. pylori infection. Non- invasive diagnostic test for Helicobacter pylori in children. Evaluation in a multicentric European study. Gut 2002;51(suppl ii):A81.

18 Imrie C, Rowland M, Bourke B, et al. Limitations to carbon 13-labeled urea breath testing for Helicobacter pylori in infants. J Pediatr $2001 ; 139: 734-7$ 